\title{
Anomalous Cepheids in the Large Magellanic Cloud
}

\section{Insight into their origin and connection with the star formation history}

\author{
G. Fiorentino ${ }^{1}$ and M. Monelli ${ }^{2,3}$ \\ 1 INAF - Osservatorio Astronomico di Bologna, via Ranzani 1, 40127 Bologna, Italy \\ e-mail: giuliana.fiorentino@oabo.inaf.it \\ 2 Instituto de Astrofísica de Canarias, Calle via Lactea s/n, 38205 La Laguna, Tenerife, Spain \\ 3 Departmento de Astrofísica, Universidad de La Laguna, 38200 La Laguna, Tenerife, Spain
}

Received 10 December 2011 / Accepted 13 February 2012

\begin{abstract}
Context. The properties of variable stars can give independent constraints on the star formation history of the host galaxy, by determining the age and metallicity of the parent population.

Aims. We investigate the pulsation properties of 84 Anomalous Cepheids (ACs) detected by the OGLE-III survey in the Large Magellanic Cloud (LMC), in order to understand the formation mechanism and the characteristics of the parent population they came from.

Methods. We used an updated theoretical pulsation scenario to derive the mass and the pulsation mode of each AC in the sample. We also used a Kolmogorov-Smirnov test to analyze the spatial distribution of the ACs, in comparison with that of other groups of variable stars, and connect their properties with the star formation history of the LMC.

Results. We find that the mean mass of ACs is $1.2 \pm 0.2 M_{\odot}$. We show that ACs do not follow the same spatial distribution of classical Cepheids. This and the difference in their period-luminosity relations further support the hypothesis that ACs are not the extension to low luminosity of classical Cepheids. The spatial distribution of ACs is also different from that of bona-fide tracers of the old population, such as RR Lyrae stars and population II Cepheids. We therefore suggest that the majority of ACs in the LMC are made of intermediate-age (1-6 Gyr), metal-poor single stars. Finally, we investigate the relation between the frequency of ACs and the luminosity of the host galaxy, disclosing that purely old systems follow a very tight relation and that galaxies with strong intermediate-age and young star formation tend to have an excess of ACs, in agreement with their hosting ACs formed via both single and binary star channels.
\end{abstract}

Key words. Magellanic Clouds - Galaxy: stellar content - stars: variables: Cepheids

\section{Introduction}

Anomalous Cepheids (ACs) are short-period variable stars (from a few hours to two days), located on the colour-magnitude diagram $\sim 1$ mag above the horizontal branch, at colours similar to those of RR Lyrae stars (Bono et al. 1997). Their properties can be explained by assuming that they are relatively massive $\left(1-2 M_{\odot}\right)$, core-He burning stars in a very low-metallicity regime that ignite helium under partial electron-degeneracy conditions $(Z \lesssim 0.0008$, Renzini et al. 1977; Castellani \& degl'Innocenti 1995; Bono et al. 1997; Caputo et al. 2004; Fiorentino et al. 2006).

ACs have been observed in many nearby Local Group dwarf galaxies that were surveyed for short time variability, independently of the morphological type. Typically, nearby dwarfs host a few of them. They have been observed in purely old systems (age $\gtrsim 9-10 \mathrm{Gyr}$ ), such as in the dwarf spheroidal (dSph) satellites Sculptor (3 ACs, Smith \& Stryker 1986; Kaluzny et al. 1995), Sextans (6, Mateo et al. 1995), LeoII (4, Siegel \& Majewski 2000), Ursa Minor (7, Nemec et al. 1988), Draco (10, Harris et al. 1998; Kinemuchi et al. 2008), and also in isolated dSphs, like Cetus (8, Bernard et al. 2009; Monelli et al. 2012),

\footnotetext{
* Based on observations released by the Optical Gravitational Lensing Experiment (OGLE-III).
}

and Tucana (6, Bernard et al. 2009). However, they have also been found both in $\mathrm{dSph}$ galaxies with a large intermediate-age population (hereinafter, 1-6 Gyr), such as Fornax (17, Bersier \& Wood 2002), Carina (15, Dall'Ora et al. 2003), and LeoI (15, Hodge \& Wright 1978) and in gas-rich dwarfs such as Phoenix (12, Gallart et al. 2004b). The cases of Leo A and NGC 6822 are not as clear, for the coexistence of ACs and short-period classical Cepheids (Hoessel et al. 1994; Dolphin et al. 2002; Baldacci et al. 2005). Furthermore, some have been identified in four satellites of M 31 (Pritzl et al. 2002, 2004, 2005) for which the star formation history ( $\mathrm{SFH}$ ) and the population content is still uncertain. Recently, three ACs have been proposed as candidates in the ultrafaint dwarf CVenI (Kuehn et al. 2008), However, nearby galaxies, especially the spheroidal satellites of the Milky Way, typically occupy large areas of the sky, so that probably the actual census is far from complete. On the other hand, ACs are very rare in globular clusters: so far, only one candidate has been confirmed in the metal-poor $([\mathrm{Fe} / \mathrm{H}] \sim-2$ dex) NGC 5466 (Zinn $\&$ Dahn 1976), and a few others have been suggested (Corwin et al. 1999; Arellano Ferro et al. 2008; Kuehn et al. 2011).

The largest sample of ACs in nearby systems (83) has been collected for the LMC in the framework of the OGLE-III project (Optical Gravitational Lensing Experiment, Udalski et al. 2008). In a series of papers devoted to the analysis of thousands 
of variable stars in the LMC (classical Cepheids, Soszyński et al. 2008b; ACs and population II Cepheids, Soszyński et al. 2008c; RR Lyrae, Soszyński et al. 2009a; long-period variables, Soszyński et al. 2009b), the OGLE-III experiment released the $V, I$ photometry, pulsation properties, and well-sampled light curves for variables with periods from a few hours to hundreds of days. In this paper, we take advantage of this rich data base to investigate the nature of ACs within the global picture depicted by the SFH and chemical evolution available nowadays (e.g. Carrera et al. 2008; Harris \& Zaritsky 2009; Saha et al. 2010; Cioni et al. 2011; Carrera et al. 2011) for the LMC.

In fact, even though ACs identify a population of stars in a well-defined mass range, the origin of their progenitors is still being debated. If they are the result of the evolution of single stars, then they trace a relatively young event in the star formation, which occurred from $\sim 1$ to $6 \mathrm{Gyr}$ ago (Demarque \& Hirshfeld 1975; Norris \& Zinn 1975; Castellani \& degl'Innocenti 1995; Caputo et al. 1999). On the other hand, it has been proposed that they are the evolution of blue straggler stars (BSS), formed via mass-exchange in binary systems (Renzini et al. 1977; Sills et al. 2009) that survived in lowdensity environments, so ACs are tracers of the old, metal-poor population. The discovery of $83 \mathrm{ACs}$ in a relatively metal-rich $(Z=0.008)$ environment such as that of the LMC makes this picture even more puzzling.

In this work, we present the approach based on pulsation models built ad hoc for this class of variable stars (Marconi et al. 2004; Caputo et al. 2004; Fiorentino et al. 2006) to constrain the pulsation mass of individual stars. This, in turn, can be related to the SFH of the host system, as already successfully done to all the ACs discovered so far in nearby galaxies (Caputo et al. 2004; Monelli et al. 2012; Fiorentino et al. 2012).

This paper is organised as follows. In Sect. 2 we give a full description of the sample selected from the OGLE-III survey of the LMC and of the Fourier analysis needed to measure the pulsation amplitude in $V$-band. In Sect. 3 we compare the OGLEIII data with the theoretical boundaries of the instability strip (Marconi et al. 2004). In Sect. 4, we describe the theoretical method used to simultaneously constrain the pulsation modes and the masses. Section 5 presents the radial distribution of different samples of variable stars in the LMC. The discussion and the conclusions close the paper.

\section{The OGLE-III sample}

We selected the 83 ACs identified in the short period Cepheids catalogue presented in Soszyński et al. (2008c). The classification of the variable type is mostly based on the period-luminosity relation (PL), as detailed in Soszyński et al. (2008a,c). In particular, the Wesenheit ${ }^{1}$ index is a good diagnostic, because it includes the colour information (and therefore the temperature) of the star without being affected by the reddening evaluation. Using this plane, it is straightforward for separating Cepheids (classical and anomalous) from population II Cepheids (hereinafter P2C, which include BL Her, W Vir, and RV Tau), because the latter have, at a given period, significantly fainter magnitudes (Fiorentino et al. 2006, and references therein). From the Wesenheit plane, it clearly emerges that anomalous and classical Cepheids occupy different regions. In particular, ACs are located at an intermediate position between classical Cepheids and P2C. However, the unambiguous distinction between anomalous and

\footnotetext{
1 The Wesenheit index is defined as $W=V-2.54 \times(V-I)$, where $V$ and $I$ are the apparent magnitudes.
}

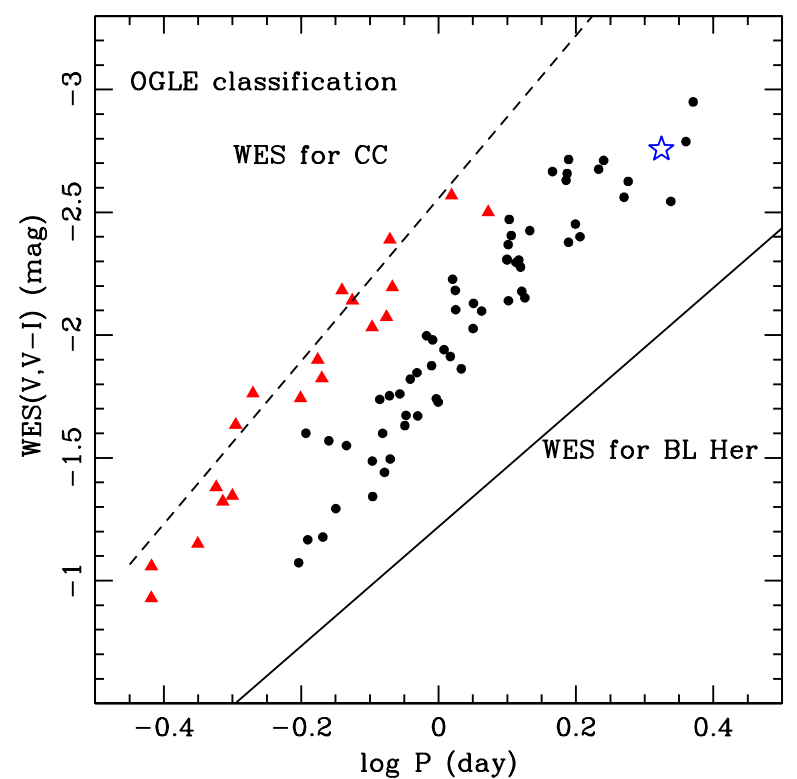

Fig. 1. Wesenheit $(V, V-I)$ plane. Dots and triangles represent fundamental and first-overtone anomalous Cepheids as defined by OGLEIII (see Soszyński et al. 2008c). The star represents one P2C candidate from OGLE-III that we have included in our sample. The solid line represents the theoretical prediction for BL Her type stars from Di Criscienzo et al. (2007), while the dashed line is the observed Wesenheit relation for fundamental-mode classical Cepheids (Soszyński et al. 2008a).

classical Cepheids is hampered by the fact that the first-overtone (FO) AC fall on the extension of the period-luminosity relation to shorter periods of the fundamental (F) classical Cepheids (period $\lesssim 2.5$ d Caputo et al. 2004; Soszyński et al. 2008c). For these stars, the shape of the light curve is the most efficient diagnostic to separate the two groups. This picture is even more complicated by certain degeneracy existing with the RR Lyrae stars as well. Therefore, while ACs with periods longer than $1 \mathrm{~d}$ can be safely identified, in the short-period regime $(<1 \mathrm{~d})$, brighter RR Lyrae stars and ACs can overlap on the period-magnitude plane. Moreover, the intrinsic similarities between the light curves of these two groups make the distinction uncertain.

The final classification from OGLE-III includes 83 ACs. On the basis of their location in the Wesenheit plane, 62 were classified as F and 21 as FO pulsators. We discuss the importance of the mode classification more fully in Sect. 4. To this sample, we added one star classified by OGLE-III as P2C (namely V-166), because it shares the pulsation properties of ACs, as we discuss in the next section. Figure 1 shows the $(V, V-I)$ Wesenheit relation for the 84 stars. For comparison, we also show the theoretical relation for BL Her stars given by Di Criscienzo et al. (2007), which are the subsample of the P2C class with a short period $(P \leq 4 \mathrm{~d})$. The Wesenheit relation for fundamental-mode classical Cepheids has been taken from Soszyński et al. (2008a).

In Table 1 we summarise some of the main properties provided by the OGLE-III catalogue: the first eight columns give, for each variable star, the identification number, the position (right ascension and declination), the mean $V$ and $I$ magnitudes, the period, the amplitude in the $I$ band $\left(A_{I}\right)$, and the mode classification. The $V$-band amplitude, $A_{V}$, was not given because the number of phase points in the $V$-band (27 to 138 ) is typically a factor $\sim 10$ smaller than in $I$ (319 to 1299). Nevertheless, the minimum number of data points in the $V$ band is 27 epochs, ensuring well-sampled light curves. For this reason, we performed 
the Fourier analysis on $V$-band photometry to give an independent estimation of the pulsation parameter and provide the amplitude $A_{V}$. Taking advantage of the accurate periods given from OGLE-III catalogue, we estimated $A_{V}$ using the GRATIS code (developed by Montegriffo; see Fiorentino et al. 2010, and reference therein). The results are reported in Cols. 9 and 10 of Table 1. The comparison with the OGLE-III mean magnitude reveals that this difference $\Delta\langle V\rangle$ is always less than about $0.05 \mathrm{mag}$. The total error budget on our evaluation gives $\sigma\left(A_{V}\right) \lesssim$ $0.1 \mathrm{mag}$, thus perfectly suitable for the goals of the present analysis. The star $V-82$ was excluded because no $V$-band photometry is available.

\section{Comparison with theoretical predictions}

To properly compare theory and observations, we need to account for the distance modulus and the reddening of the host galaxy. The distance modulus to the LMC has been extensively discussed in the literature, since this galaxy is the cornerstone of the distance scale. We adopt here $\mu_{0}=18.50 \mathrm{mag}$, as given by a recent estimate using Red Clump stars observed in optical and near-IR (Fiorentino et al. 2011). This agrees with many of the measurements derived by independent methods, including classical Cepheids (Bono et al. 2002), RR Lyrae stars (Catelan \& Cortés 2008), planetary nebulae luminosity function (Reid $\&$ Parker 2010), the red clump, and the tip of the red giant branch (Romaniello et al. 2000). The AC sample distributed across the whole area surveyed by OGLE-III, we cannot adopt a unique reddening value for all the variables. To correct the magnitude of each star for the appropriate extinction value, we used the reddening map recently derived by Haschke et al. (2011) using red clump and RR Lyrae stars from the same OGLE-III release. In particular, we assumed the reddening value, $E(V-I)$, for the closest position available for each individual AC. This is reported in Col. 11 of Table 1 . The mean reddening is $E(V-I) \sim$ $0.08 \mathrm{mag}$, and only one star has a value higher than $0.2 \mathrm{mag}$.

In Fig. 2 we show the comparison between the theoretical prediction for the boundaries of the pulsation instability strip (Fiorentino et al. 2006) and the sample of ACs. The top and bottom panels show, as a function of the logarithm of the period, the absolute mean colour $\left(\left\langle M_{V}\right\rangle-\left\langle M_{I}\right\rangle\right)$ and the absolute mean $\left\langle M_{V}\right\rangle$ magnitude, respectively. The agreement between theory and observations is good in both cases and for all the objects (the symbols are the same as in Fig. 1). The star, classified by OGLE-III as population II Cepheids, seems to fully agree with AC observational properties. We note that there are few outliers in the upper panel. This is possibly due to the uncertainty in the reddening determination, with directly affects the true colour of stars. Nevertheless, this comparison suggests that the correction for reddening applied is satisfactory for most of the objects.

\section{Constraining masses and pulsation modes}

AC stars follow well-defined relations, which are the periodmass-amplitude relation (PMA) and the mass dependent periodluminosity-colour relation (PMC). Here, it is worth mentioning that this theoretical scenario was used for classical Cepheids to constrain pulsation masses with very high precision $(1 \%)$ and returned values in good agreement with dynamical masses (e.g. Pietrzyński et al. 2010; Pietrzynski et al. 2011; Cassisi \& Salaris 2011). In particular, it is possible to estimate individual

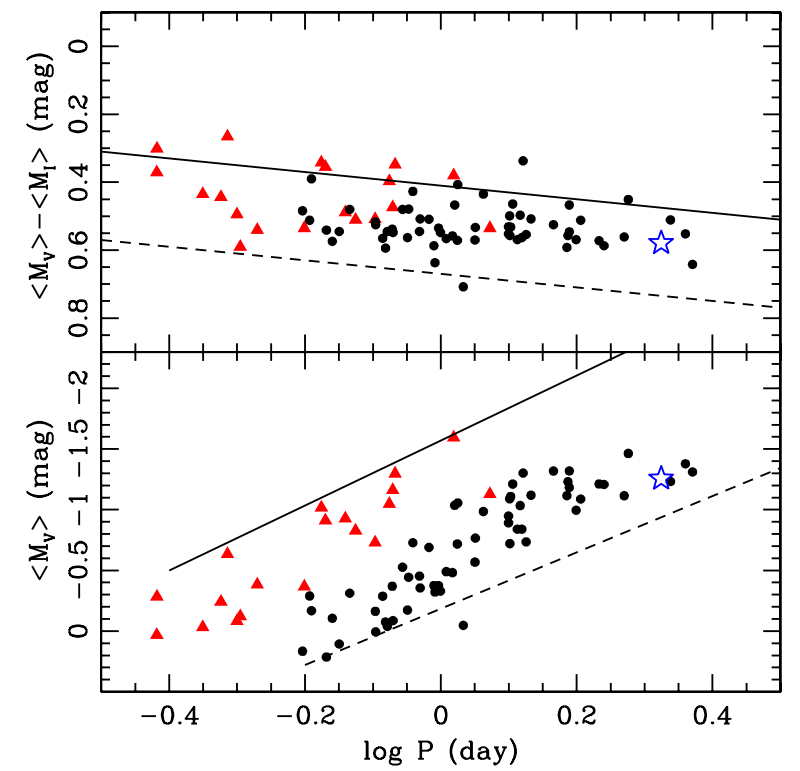

Fig. 2. Comparison between theoretical models and OGLE-III observations. The distance modulus assumed for LMC is $\mu_{0}=18.50 \mathrm{mag}$ (Fiorentino et al. 2011), and the individual reddening values are assumed using the reddening map given by Haschke et al. (2011) (see text for details). The coding of the colours is the same as in previous figures. Top $-\left(\left\langle M_{V}\right\rangle-\left\langle M_{I}\right\rangle\right)_{0}$ colour vs. period $(\log P)$ diagram are compared with theoretical blue (solid line) and red (dashed line) edges of the instability strip (Marconi et al. 2004); bottom - absolute magnitude $\left(M_{V}\right)$ vs. period $(\log P)$ plane compared with the theoretical first overtone blue (solid line) and the fundamental red (dashed line) edges of the instability strip.

masses once distance and reddening are known by applying the following equations, adapted from Marconi et al. (2004):

$$
\begin{aligned}
M_{\mathrm{F}, \mathrm{PMA}} / M_{\odot} & =10^{\left(0.013-0.53\left\langle M_{V}\right\rangle-1.3 \log P-0.244 A_{V}\right)} \\
M_{\mathrm{F}, \mathrm{PMC}} / M_{\odot} & =10^{\left(-0.97-0.53\left\langle M_{V}\right\rangle-1.55 \log P+1.44\left(\left\langle M_{V}\right\rangle-\left\langle M_{I}\right\rangle\right)\right)} \\
M_{\mathrm{FO}, \mathrm{PMC}} / M_{\odot} & =10^{\left(-1.29-0.58\left\langle M_{V}\right\rangle-1.79 \log P+1.54\left(\left\langle M_{V}\right\rangle-\left\langle M_{I}\right\rangle\right)\right)},
\end{aligned}
$$

where $M_{V}, M_{I}$ are the absolute intensity-weighted mean $V, I$ magnitudes, $P$ is the period, and $A_{V}$ is the amplitude in the $V$ band. In this work, we assumed reddening law from Cardelli et al. (1989). The formal uncertainty on the derived masses is $5 \%$ using the PMC and 15\% using the PMA (Marconi et al. 2004). It is worth mentioning that the comparison of the masses derived with a different relation is independent of the distance modulus adopted, but it does depend on the reddening correction applied. Assuming the OGLE-III classification, we estimated the mass of each star, which is reported in Table 1 . We provide the mean mass from Eqs. (1) and (2) in the case of F, and the mass from Eq. (3) for FO. For three objects, we were not able to estimate a reliable mass, e.g. the mass estimated for the FU V-24 and V59 with Eqs. (1) and (2) shows high discrepancies, and the mass estimated for FO V-28 is too high $\left(\sim 2.3 M_{\odot}\right)$ for an AC.

A method is discussed in Marconi et al. (2004) which uses the previous relations to simultaneously constrain the pulsation mode and the mass of the star. This method is based on the fact that the PMA relation is only valid for the F pulsator, whereas the PMC exists for both pulsation modes. Therefore, whenever the PMA and the PMC for F pulsators give consistent mass, this is a robust indication that the star is actually pulsating in the fundamental mode. The correct mode classification is important because it affects the mass determination. For the same luminosity and colour, we expect $\mathrm{F}$ pulsator to be more massive than FO pulsators. However, the usual diagnostics used for 
Table 1. Anomalous Cepheids from the OGLE-III sample, including 1 star (last row) classified by OGLE-III as P2C (see text for details).

\begin{tabular}{|c|c|c|c|c|c|c|c|c|c|c|c|c|c|}
\hline \multicolumn{8}{|c|}{ OGLE-III } & \multicolumn{6}{|c|}{ This work } \\
\hline ID & $\begin{array}{c}\text { RA } \\
\text { J2000 }\end{array}$ & $\begin{array}{c}\text { Dec } \\
\text { J2000 }\end{array}$ & $\begin{array}{c}V \\
\text { mag }\end{array}$ & $\begin{array}{c}I \\
\text { mag }\end{array}$ & $\begin{array}{l}P \\
\mathrm{~d}\end{array}$ & $\begin{array}{c}A_{I} \\
\text { mag }\end{array}$ & mode $\mathrm{OGLE}$ & $\begin{array}{l}\langle\langle V\rangle \\
\text { mag }\end{array}$ & $\begin{array}{c}A_{V} \\
\text { mag }\end{array}$ & $\begin{array}{c}E(V-I) \\
\text { mag }\end{array}$ & $\begin{array}{l}M_{\mathrm{F}} \\
M_{\odot}\end{array}$ & $\begin{array}{c}M_{\mathrm{FO}} \\
M_{\odot}\end{array}$ & mode $_{\text {mass }}$ \\
\hline 001 & $04: 37: 18.96$ & $-69: 49: 11.5$ & 18.676 & 18.018 & 0.8502326 & 0.652 & $\mathrm{~F}$ & 18.692 & 0.843 & 0.110 & 0.9 & - & $\mathrm{F}$ \\
\hline 002 & $04: 41: 56.22$ & $-66: 51: 59.1$ & 18.268 & 17.621 & 0.9766401 & 0.511 & $\mathrm{~F}$ & 18.264 & 0.838 & 0.060 & 1.1 & - & $\mathrm{F}$ \\
\hline 003 & $04: 43: 16.03$ & $-69: 07: 57.6$ & 18.794 & 18.313 & 0.3817797 & 0.529 & FO & 18.787 & 0.880 & 0.110 & (1.8) & 1.0 & $\mathrm{~F}^{* *}$ \\
\hline 004 & $04: 47: 21.63$ & $-70: 22: 12.0$ & 17.719 & 17.018 & 1.8618275 & 0.637 & $\mathrm{~F}$ & 17.739 & 0.959 & 0.140 & 1.0 & - & $\mathrm{F}$ \\
\hline 005 & $04: 51: 11.62$ & $-69: 00: 33.0$ & 18.526 & 17.858 & 0.9321588 & 0.243 & $\mathrm{~F}$ & 18.527 & 0.382 & 0.160 & 1.2 & - & $\mathrm{F}$ \\
\hline 006 & $04: 52: 34.51$ & $-69: 43: 27.5$ & 17.671 & 17.057 & 0.8495758 & 0.389 & FO & 17.671 & 0.682 & 0.140 & - & 1.7 & FO \\
\hline 007 & $04: 57: 31.48$ & $-70: 15: 53.6$ & 18.247 & 17.688 & 0.8963988 & 0.703 & $\mathrm{~F}$ & 18.143 & 1.180 & 0.080 & 1.1 & - & $\mathrm{F}$ \\
\hline 008 & $04: 58: 24.59$ & $-71: 05: 11.5$ & 17.911 & 17.300 & 0.7490681 & 0.262 & $\mathrm{FO}$ & 17.912 & 0.425 & 0.100 & - & 1.6 & FO \\
\hline 009 & $04: 58: 51.29$ & $-67: 44: 23.9$ & 17.913 & 17.344 & 0.8000803 & 0.366 & FO & 17.896 & 0.597 & 0.060 & $(2.2)$ & 1.2 & $\mathrm{~F}^{* *}$ \\
\hline 010 & 04:59:00.10 & $-68: 14: 01.1$ & 18.723 & 18.068 & 0.8342013 & 0.705 & $\mathrm{~F}$ & 18.628 & 1.082 & 0.110 & 0.8 & - & $\mathrm{F}$ \\
\hline 011 & 04:59:38.09 & $-70: 37: 45.5$ & 18.254 & 17.671 & 0.9985904 & 0.561 & $\mathrm{~F}$ & 18.299 & 0.843 & 0.035 & 1.0 & - & $\mathrm{F}$ \\
\hline 012 & $04: 59: 49.43$ & $-69: 33: 12.4$ & 18.663 & 17.969 & 0.8289912 & 0.445 & $\mathrm{~F}$ & 18.679 & 0.719 & 0.100 & 1.1 & - & $\mathrm{F}$ \\
\hline 013 & $04: 59: 56.62$ & $-70: 42: 24.9$ & 18.488 & 17.963 & 0.5009227 & 0.446 & FO & 18.480 & 0.737 & 0.030 & (1.8) & 1.1 & $\mathrm{~F}^{* *}$ \\
\hline 014 & 05:00:08.26 & $-67: 54: 04.3$ & 17.241 & 16.639 & 2.2913456 & 0.580 & $\mathrm{~F}$ & 17.263 & 0.787 & 0.050 & 1.1 & - & $\mathrm{F}$ \\
\hline 015 & 05:00:59.32 & $-69: 33: 24.9$ & 17.587 & 16.962 & 1.1808787 & 0.383 & FO & 17.578 & 0.540 & 0.090 & $(2.2)$ & 1.1 & $\mathrm{~F}^{* *}$ \\
\hline 016 & $05: 01: 36.69$ & $-67: 51: 33.1$ & 17.448 & 16.926 & 1.5456697 & 0.763 & $\mathrm{~F}$ & 17.450 & 1.197 & 0.055 & 1.2 & - & $\mathrm{F}$ \\
\hline 017 & $05: 02: 03.13$ & $-68: 09: 30.4$ & 18.190 & 17.585 & 0.9299953 & 0.550 & F & 18.185 & 0.897 & 0.060 & 1.2 & - & $\mathrm{F}$ \\
\hline 018 & $05: 02: 36.36$ & $-69: 40: 12.2$ & 18.213 & 17.562 & 1.0185719 & 0.476 & $\mathrm{~F}$ & 18.229 & 0.798 & 0.085 & 1.2 & - & $\mathrm{F}$ \\
\hline 019 & 05:03:17.71 & $-68: 49: 33.5$ & 17.891 & 17.414 & 0.9094057 & 0.839 & $\mathrm{~F}$ & 17.895 & 1.295 & 0.050 & 1.3 & - & $\mathrm{F}$ \\
\hline 020 & $05: 05: 54.54$ & $-70: 43: 30.3$ & 18.359 & 17.998 & 0.3819013 & 0.491 & FO & 18.362 & 0.768 & 0.060 & - & 1.2 & FO \\
\hline 021 & 05:06:37.49 & $-68: 23: 40.3$ & 17.827 & 17.188 & 1.2958429 & 0.750 & $\mathrm{~F}$ & 17.821 & 1.166 & 0.070 & 1.2 & - & $\mathrm{F}$ \\
\hline 022 & $05: 06: 45.08$ & $-70: 52: 44.7$ & 18.378 & 17.796 & 0.6409626 & 0.484 & $\mathrm{~F}$ & 18.400 & 0.754 & 0.070 & 1.7 & - & $\mathrm{F}$ \\
\hline 023 & $05: 07: 52.42$ & $-68: 50: 28.8$ & 17.763 & 17.194 & 0.7234331 & 0.400 & FO & 17.761 & 0.650 & 0.080 & - & 1.8 & FO \\
\hline 024 & $05: 08: 44.05$ & $-68: 46: 01.2$ & 17.986 & 17.679 & 0.7944643 & 0.471 & $\mathrm{~F}$ & 18.000 & 0.747 & 0.110 & - & - & n.c. \\
\hline 025 & 05:09:55.67 & $-70: 03: 08.2$ & 18.413 & 17.904 & 0.4744618 & 0.354 & FO & 18.417 & 0.574 & 0.065 & (2.3) & 1.3 & $\mathrm{~F}^{* *}$ \\
\hline 026 & $05: 10: 42.62$ & $-68: 48: 19.6$ & 17.483 & 16.816 & 1.7387452 & 0.607 & $\mathrm{~F}$ & 17.483 & 0.987 & 0.080 & 1.3 & - & $\mathrm{F}$ \\
\hline 027 & $05: 10: 45.77$ & $-67: 10: 05.6$ & 17.558 & 16.956 & 1.2670644 & 0.973 & $\mathrm{~F}$ & 17.550 & 1.786 & 0.070 & 1.4 & $(1.0)$ & FO* \\
\hline 028 & $05: 13: 10.02$ & $-68: 46: 11.9$ & 18.322 & 17.574 & 0.5992535 & 0.332 & FO & 18.320 & 0.672 & 0.060 & - & - & n.c. \\
\hline 029 & $05: 13: 33.58$ & $-67: 33: 42.2$ & 18.745 & 18.120 & 0.8017029 & 0.441 & $\mathrm{~F}$ & 18.745 & 0.659 & 0.100 & 0.9 & - & $\mathrm{F}$ \\
\hline 030 & $05: 15: 07.61$ & $-68: 04: 21.4$ & 17.685 & 17.258 & 0.6670589 & 0.538 & FO & 17.685 & 0.877 & 0.085 & - & 1.4 & FO \\
\hline 031 & $05: 15: 32.98$ & $-68: 10: 29.4$ & 17.727 & 17.215 & 0.8397205 & 0.401 & FO & 17.728 & 0.689 & 0.115 & - & 1.1 & FO \\
\hline 032 & $05: 15: 56.14$ & $-69: 01: 29.1$ & 17.780 & 17.167 & 1.3160220 & 0.425 & $\mathrm{~F}$ & 17.778 & 0.651 & 0.050 & 1.3 & - & $\mathrm{F}$ \\
\hline 033 & $05: 16: 08.01$ & $-70: 39: 16.5$ & 17.308 & 16.616 & 2.3470013 & 0.186 & $\mathrm{~F}$ & 17.305 & 0.287 & 0.050 & 1.3 & - & $\mathrm{F}$ \\
\hline 034 & $05: 17: 11.15$ & $-69: 58: 33.1$ & 18.474 & 17.874 & 0.7342975 & 0.312 & $\mathrm{~F}$ & 18.477 & 0.561 & 0.120 & 1.4 & - & F \\
\hline 035 & $05: 18: 22.19$ & $-69: 03: 38.4$ & 18.658 & 18.143 & 0.4460486 & 0.508 & FO & 18.658 & 0.793 & 0.080 & (1.8) & 1.1 & $\mathrm{~F}^{* *}$ \\
\hline 036 & $05: 18: 58.84$ & $-69: 26: 47.8$ & 17.787 & 17.160 & 1.2579824 & 0.281 & $\mathrm{~F}$ & 17.787 & 0.406 & 0.075 & 1.6 & - & $\mathrm{F}$ \\
\hline 037 & $05: 19: 16.66$ & $-70: 11: 58.4$ & 17.743 & 17.132 & 1.2577403 & 0.794 & $\mathrm{~F}$ & 17.703 & 1.099 & 0.080 & 1.4 & - & $\mathrm{F}$ \\
\hline 038 & $05: 19: 59.97$ & $-71: 45: 23.7$ & 17.908 & 17.294 & 1.3352133 & 0.518 & $\mathrm{~F}$ & 17.907 & 0.797 & 0.060 & 1.1 & - & $\mathrm{F}$ \\
\hline 039 & $05: 20: 44.46$ & $-69: 47: 46.5$ & 18.245 & 17.660 & 0.9924071 & 0.621 & $\mathrm{~F}$ & 18.249 & 0.908 & 0.050 & 1.0 & - & $\mathrm{F}$ \\
\hline 040 & $05: 21: 13.22$ & $-70: 34: 20.7$ & 18.037 & 17.433 & 0.9605775 & 0.513 & $\mathrm{~F}$ & 18.040 & 0.803 & 0.095 & 1.5 & - & $\mathrm{F}$ \\
\hline 041 & $05: 21: 14.35$ & $-70: 29: 39.5$ & 18.200 & 17.625 & 0.8781418 & 0.548 & $\mathrm{~F}$ & 18.203 & 0.854 & 0.095 & 1.3 & - & F \\
\hline 042 & $05: 23: 34.60$ & $-69: 10: 58.2$ & 18.715 & 17.897 & 1.0790357 & 0.671 & $\mathrm{~F}$ & 18.705 & 1.019 & 0.110 & 0.8 & $(0.6)$ & FO* \\
\hline 043 & $05: 24: 35.40$ & $-68: 48: 22.6$ & 18.593 & 17.913 & 0.5064704 & 0.356 & FO & 18.593 & 0.542 & 0.090 & - & 1.6 & $\mathrm{FO}$ \\
\hline 044 & $05: 25: 54.11$ & $-69: 26: 52.9$ & 17.609 & 17.052 & 1.3085090 & 0.913 & $\mathrm{~F}$ & 17.596 & 1.398 & 0.060 & 1.2 & - & $\mathrm{F}$ \\
\hline 045 & $05: 26: 24.68$ & $-68: 57: 55.0$ & 18.976 & 18.325 & 0.6784311 & 0.453 & $\mathrm{~F}$ & 18.977 & 0.804 & 0.110 & 0.9 & - & $\mathrm{F}$ \\
\hline 046 & $05: 26: 27.17$ & $-69: 58: 57.0$ & 17.851 & 17.264 & 1.2637169 & 0.592 & $\mathrm{~F}$ & 17.849 & 0.931 & 0.030 & 1.1 & - & $\mathrm{F}$ \\
\hline 047 & $05: 27: 05.27$ & $-71: 23: 33.4$ & 17.482 & 16.881 & 2.1779846 & 0.493 & $\mathrm{~F}$ & 17.475 & 0.769 & 0.090 & 0.9 & - & $\mathrm{F}$ \\
\hline 048 & $05: 27: 12.12$ & $-69: 37: 19.6$ & 17.324 & 16.718 & 1.5458930 & 0.751 & $\mathrm{~F}$ & 17.321 & 1.209 & 0.060 & 1.6 & - & $\mathrm{F}$ \\
\hline 049 & $05: 28: 03.57$ & $-69: 39: 15.2$ & 18.451 & 18.011 & 0.6447960 & 0.451 & $\mathrm{~F}$ & 18.455 & 0.682 & 0.050 & 1.2 & - & $\mathrm{F}$ \\
\hline 050 & $05: 28: 57.71$ & $-70: 07: 15.5$ & 17.049 & 16.609 & 1.0446912 & 0.383 & FO & 17.053 & 0.591 & 0.060 & - & 1.5 & FO \\
\hline 051 & $05: 30: 14.24$ & $-68: 42: 31.1$ & 18.845 & 18.200 & 0.7086059 & 0.318 & $\mathrm{~F}$ & 18.842 & 0.559 & 0.100 & 1.0 & - & $\mathrm{F}$ \\
\hline 052 & $05: 31: 01.53$ & $-70: 42: 22.2$ & 17.577 & 17.008 & 1.2625549 & 0.823 & $\mathrm{~F}$ & 17.579 & 1.309 & 0.070 & 1.4 & - & $\mathrm{F}$ \\
\hline 053 & $05: 31: 06.20$ & $-68: 43: 45.3$ & 17.299 & 16.738 & 1.8880987 & 0.721 & $\mathrm{~F}$ & 17.289 & 1.128 & 0.110 & 1.3 & - & $\mathrm{F}$ \\
\hline 054 & $05: 31: 06.72$ & $-68: 22: 29.8$ & 18.798 & 17.901 & 0.9802225 & 0.739 & $\mathrm{~F}$ & 18.776 & 1.197 & 0.260 & 1.1 & $(0.8)$ & $\mathrm{FO}^{*}$ \\
\hline 055 & $05: 31: 41.11$ & $-68: 44: 37.7$ & 17.603 & 17.011 & 1.6066649 & 0.850 & $\mathrm{~F}$ & 17.549 & 1.266 & 0.080 & 1.1 & - & $\mathrm{F}$ \\
\hline 056 & $05: 31: 49.45$ & $-70: 33: 22.6$ & 17.877 & 17.284 & 1.1240035 & 0.727 & $\mathrm{~F}$ & 17.861 & 1.070 & 0.060 & 1.3 & - & $\mathrm{F}$ \\
\hline 057 & $05: 31: 49.88$ & $-70: 46: 30.0$ & 17.455 & 16.813 & 1.7100082 & 0.686 & $\mathrm{~F}$ & 17.437 & 1.307 & 0.070 & 1.2 & - & $\mathrm{F}$ \\
\hline 058 & $05: 33: 39.38$ & $-70: 21: 28.6$ & 18.055 & 17.710 & 0.4852351 & 0.255 & FO & 18.056 & 0.321 & 0.080 & - & 1.1 & $\mathrm{FO}$ \\
\hline 059 & $05: 34: 14.81$ & $-68: 23: 43.0$ & 20.004 & 18.724 & 0.8348234 & 0.717 & $\mathrm{~F}$ & 20.011 & 1.196 & 0.100 & - & - & n.c. \\
\hline 060 & 05:36:08.89 & $-70: 37: 01.3$ & 17.527 & 16.963 & 1.2757288 & 0.942 & $\mathrm{~F}$ & 17.516 & 1.464 & 0.100 & 1.5 & - & $\mathrm{F}$ \\
\hline 061 & $05: 36: 24.32$ & $-71: 30: 39.4$ & 18.393 & 17.745 & 0.8480731 & 0.632 & $\mathrm{~F}$ & 18.389 & 1.049 & 0.110 & 1.2 & - & $\mathrm{F}$ \\
\hline 062 & $05: 36: 58.43$ & $-70: 46: 08.1$ & 17.659 & 17.162 & 1.0590929 & 0.769 & $\mathrm{~F}$ & 17.662 & 1.250 & 0.090 & 1.6 & - & $\mathrm{F}$ \\
\hline 063 & $05: 37: 54.39$ & $-69: 19: 28.7$ & 18.756 & 18.013 & 0.8930306 & 0.534 & $\mathrm{~F}$ & 18.762 & 0.849 & 0.180 & 1.0 & - & $\mathrm{F}$ \\
\hline 064 & $05: 39: 26.03$ & $-71: 24: 47.6$ & 17.619 & 17.011 & 1.3574685 & 0.837 & $\mathrm{~F}$ & 17.594 & 1.342 & 0.100 & 1.4 & - & F \\
\hline 065 & $05: 40: 03.04$ & $-70: 04: 47.8$ & 17.508 & 17.041 & 1.3215432 & 0.888 & $\mathrm{~F}$ & 17.515 & 1.386 & 0.130 & 1.3 & - & $\mathrm{F}$ \\
\hline 066 & $05: 45: 53.29$ & $-72: 01: 19.6$ & 18.233 & 17.585 & 1.0400730 & 0.570 & $\mathrm{~F}$ & 18.229 & 0.933 & 0.090 & 1.1 & - & $\mathrm{F}$ \\
\hline 067 & $05: 48: 22.07$ & $-70: 45: 49.3$ & 18.451 & 17.786 & 0.8209206 & 0.629 & $\mathrm{~F}$ & 18.407 & 1.082 & 0.100 & 1.2 & - & $\mathrm{F}$ \\
\hline 068 & $05: 51: 09.02$ & $-70: 45: 42.6$ & 18.809 & 18.265 & 0.6256447 & 0.746 & $\mathrm{~F}$ & 18.781 & 1.095 & 0.060 & 0.9 & - & F \\
\hline 069 & $05: 53: 31.91$ & $-71: 32: 44.8$ & 17.460 & 16.823 & 1.5384361 & 0.674 & $\mathrm{~F}$ & 17.454 & 1.134 & 0.080 & 1.5 & - & $\mathrm{F}$ \\
\hline 070 & $05: 53: 43.23$ & $-69: 17: 06.0$ & 18.370 & 17.735 & 0.6293707 & 0.271 & FO & 18.354 & 0.471 & 0.100 & $(2.2)$ & 1.3 & $\mathrm{~F}^{* *}$ \\
\hline 071 & $05: 54: 43.24$ & $-70: 10: 16.1$ & 17.755 & 17.330 & 0.6762087 & 0.406 & FO & 17.755 & 0.687 & 0.070 & - & 1.2 & FO \\
\hline
\end{tabular}


Table 1. continued.

\begin{tabular}{|c|c|c|c|c|c|c|c|c|c|c|c|c|c|}
\hline \multicolumn{8}{|c|}{ OGLE-III } & \multicolumn{6}{|c|}{ This work } \\
\hline ID & $\begin{array}{c}\text { RA } \\
\text { J2000 }\end{array}$ & $\begin{array}{c}\text { Dec } \\
\text { J2000 }\end{array}$ & $\begin{array}{c}V \\
\mathrm{mag}\end{array}$ & $\begin{array}{c}I \\
\mathrm{mag}\end{array}$ & $\begin{array}{l}P \\
\mathrm{~d}\end{array}$ & $\begin{array}{c}A_{I} \\
\text { mag }\end{array}$ & mode $_{\text {OGLE }}$ & $\begin{array}{l}\langle V\rangle \\
\mathrm{mag}\end{array}$ & $\begin{array}{c}A_{V} \\
\text { mag }\end{array}$ & $\begin{array}{c}E(V-I) \\
\text { mag }\end{array}$ & $\begin{array}{l}M_{\mathrm{F}} \\
M_{\odot}\end{array}$ & $\begin{array}{c}M_{\mathrm{FO}} \\
M_{\odot}\end{array}$ & mode $_{\text {mass }}$ \\
\hline 072 & $05: 59: 08.23$ & $-68: 24: 01.2$ & 17.535 & 17.038 & 1.0482162 & 0.901 & $\mathrm{~F}$ & 17.530 & 1.385 & 0.030 & 1.6 & - & $\bar{F}$ \\
\hline 073 & 06:00:04.99 & $-70: 44: 59.8$ & 17.396 & 16.781 & 1.4650553 & 0.799 & $\mathrm{~F}$ & 17.389 & 1.008 & 0.090 & 1.7 & - & $\mathrm{F}$ \\
\hline 074 & $06: 02: 45.18$ & $-70: 45: 31.2$ & 17.551 & 16.889 & 1.5332247 & 0.322 & $\mathrm{~F}$ & 17.549 & 0.498 & 0.070 & 1.7 & - & $\mathrm{F}$ \\
\hline 075 & $06: 03: 33.33$ & $-68: 27: 41.4$ & 18.490 & 17.876 & 0.6920857 & 0.328 & $\mathrm{~F}$ & 18.446 & 0.556 & 0.040 & 1.4 & - & F \\
\hline 076 & 06:04:33.02 & $-70: 57: 55.8$ & 17.671 & 17.032 & 1.5818249 & 0.563 & $\mathrm{~F}$ & 17.669 & 0.874 & 0.070 & 1.2 & - & $\mathrm{F}$ \\
\hline 077 & $06: 04: 35.73$ & $-71: 40: 35.8$ & 18.099 & 17.459 & 1.1224977 & 0.312 & F & 18.098 & 0.502 & 0.070 & 1.3 & - & $\mathrm{F}$ \\
\hline 078 & $06: 06: 58.20$ & $-72: 52: 08.7$ & 17.417 & 16.979 & 0.8565557 & 0.391 & FO & 17.420 & 0.659 & 0.090 & - & 1.3 & $\mathrm{FO}$ \\
\hline 079 & $06: 07: 02.01$ & $-69: 31: 55.2$ & 17.634 & 17.149 & 1.1551702 & 0.932 & $\mathrm{~F}$ & 17.665 & 1.482 & 0.050 & 1.2 & - & $\mathrm{F}$ \\
\hline 080 & $06: 09: 35.12$ & $-70: 10: 42.1$ & 17.997 & 17.336 & 1.0574863 & 0.307 & $\mathrm{~F}$ & 17.995 & 0.540 & 0.090 & 1.6 & - & $\mathrm{F}$ \\
\hline 081 & 06:09:38.40 & $-69: 34: 04.1$ & 18.504 & 17.917 & 0.8008377 & 0.503 & $\mathrm{~F}$ & 18.510 & 0.874 & 0.070 & 1.0 & - & $\mathrm{F}$ \\
\hline 082 & $06: 16: 58.31$ & $-70: 52: 18.9$ & -- & 17.207 & 0.7754317 & 0.469 & - & - & - & - & - & - & n.c. \\
\hline 083 & $06: 18: 52.45$ & $-70: 51: 55.4$ & 18.236 & 17.646 & 0.5366171 & 0.497 & FO & 18.238 & 0.529 & 0.050 & - & 1.8 & $\mathrm{FO}$ \\
\hline 166 & $05: 16: 58.99$ & $-69: 51: 19.3$ & 17.696 & 16.927 & 2.1105987 & 0.267 & $\mathrm{P} 2 \mathrm{C}$ & 17.694 & 0.490 & 0.190 & 1.2 & - & $\mathrm{F}$ \\
\hline
\end{tabular}

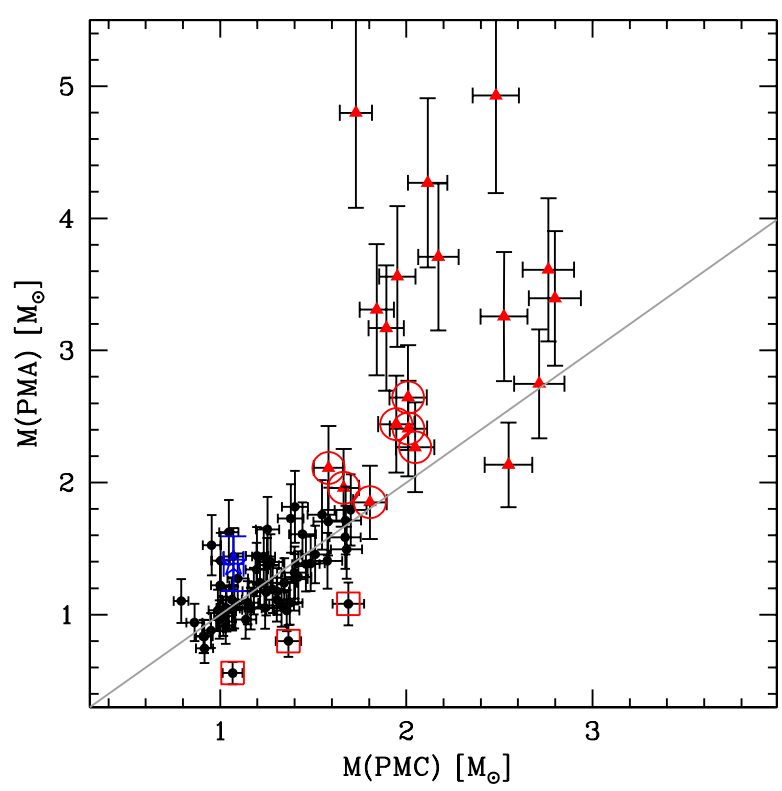

Fig. 3. Mass values for anomalous Cepheids in the LMC as derived from PMA and PMC relationships. Solid grey line shows the relation of equality. The coding of the colours is the same as in previous figures. The ACs identified as FO in OGLE but F in this work are highlighted with red open circles, while the ones classified as F in OGLE and FO in this work have been emphasised with open squares.

other classes of variable stars, such as the Bailey diagram $\left(A_{V}\right.$ vs. $\log P$ plane) or the light curve morphology, do not allow the pulsation mode to be uniquely constrained. For this reason, we applied the method given in Marconi et al. (2004) to check whether the mode classification based on the Wesenheit index (provided by the OGLE team) is consistent with theoretical pulsation predictions.

Figure 3 compares the mass values obtained using Eqs. (1) and (2). The grey line shows the relation of equality. Assuming the formal error for the theoretical relations, we classified as $\mathrm{F}$ pulsators all the stars for which the mass difference is within the sum of the two $1-\sigma$ errors. For a better identification, we have also classified $\mathrm{F}$ pulsators all those stars that did not pass the previous criterion, but show high PMC masses $\left(\gtrsim 2.5 M_{\odot}\right)$, which are unrealistic in the adopted theoretical scenario. Out of 83 stars, we then classify $66 \mathrm{~F}$ and $17 \mathrm{FO}$, as reported in the last column of Table 1 . Three stars (V-24, V-28, and V-59) present a large mass difference and also an atypical amplitude ratio, and at least in one case, a very red colour. This suggests that they might be blended sources, so they are reported as unclassified in Table 1 (n.c.). Moreover, V-166 previously identified as P2C turns out to be an AC pulsating in F mode.

The comparison with the OGLE-III classification reveals some discrepancy. Out of 19 FO for which we could estimate the pulsation mode, we only recover 12 . The remaining seven stars were classified as fundamental mode pulsators with our method, and are labelled as " $\mathrm{F}^{* *}$ " in Table 1 and highlighted in Fig. 3. For these stars, we also report, in parenthesis, the mass value corresponding to the $\mathrm{F}$ mode given by our classification. For these stars the FO mass value has a reliable value, systematically $\gtrsim 1 M_{\odot}$. Then, masses of these "F**" stars are compatible with both $\mathrm{F}$ pulsators with relatively higher mass, and FO pulsator of lower mass.

On the other hand, there are three stars, flagged with "FO*" in Table 1 and classified as FO, differently from the OGLE-III work. For these stars, the masses derived with the PMC result to be always higher than the ones derived with the PMA (less than $1 \sim M_{\odot}$ ), in disagreement with the general behaviour (Caputo et al. 2004). Their position in Fig. 3 shows that they are close to the bulk of F-mode stars.

To investigate the reason for these differences, we recalculated the masses of the 12 outliers varying the values of the reddening and the amplitude $A_{V}$ (which could be affected by blending). We found that, while modifying the amplitude has little or no effect, modifying the reddening value has a stronger effect. As an example, increasing the reddening of 0.1 changes the classification of $2 \mathrm{FO}\left(\mathrm{FO}^{*}\right.$ in table) out of 3 to $\mathrm{F}$ and $5 \mathrm{~F}$ ( $\mathrm{F}^{* *}$ in table) out of 7 to FO. This is an indication that the uncertainty on the reddening is the dominant source of error in the present analysis.

In Fig. 4 we show the Wesenheit relation, similar to Fig. 1, (top) and the $P-A_{V}$ (bottom), and we highlight in both panels the stars for which we find a different mode classification, as in Fig. 3. We note that the FO our method recovers as $\mathrm{F}$ all lie on the lower envelope of the FO relation in the Wesenheit plane, thus supporting the classification's consistency with both FO and F modes. Also, the Bailey diagram clearly shows that, as opposed to the case of RR Lyrae stars, this plane is not an efficient diagnostic to separate F and FO pulsators. In fact, while the shortest period ACs are clearly FO, while those with large amplitudes tend to be $\mathrm{F}$, there is a clear degeneracy in the low-amplitude regime, for periods with $-0.2 \lesssim \log P \lesssim 0.1 \mathrm{~d}$.

\section{Spatial distributions of variable stars}

To better understand the origin of ACs we take advantage of the extensive and complete sample of variable stars discovered by 


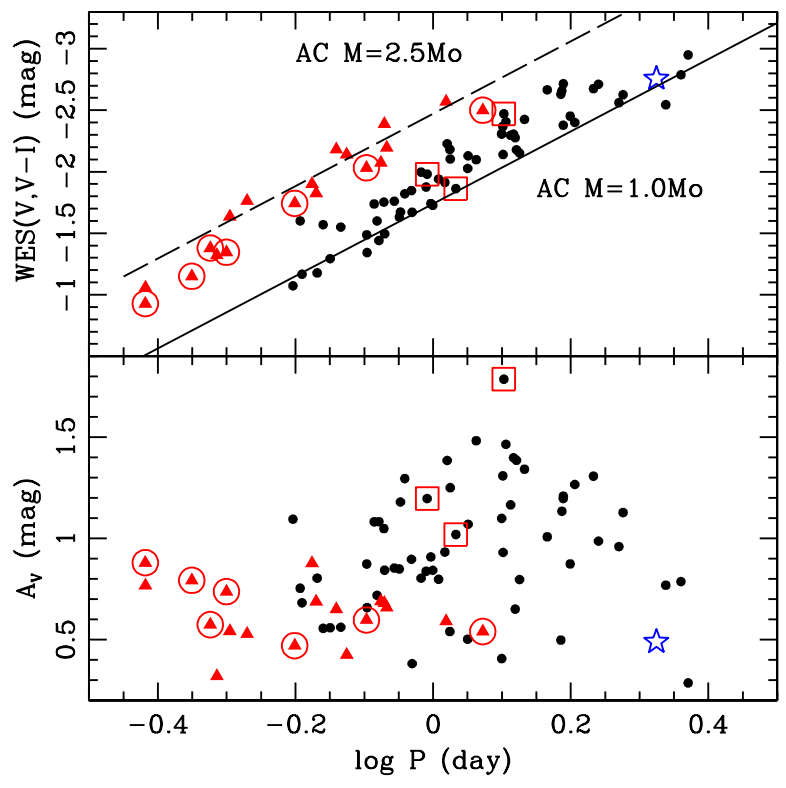

Fig. 4. Top - same as Fig. 1, but we highlight the stars for which we obtain a different mode classification as in Fig. 3. Bottom - amplitude $\left(A_{V}\right)$ vs. period $(\log P)$ diagram. The coding of the colours is the same used in Fig. 1. The amplitudes have been derived in this paper.

OGLE-III survey of the LMC, namely 22,651 RR Lyrae ( $a b$ and $c$ type), $203 \mathrm{P} 2 \mathrm{C}$, and 3,087 young classical Cepheids (F and FO only). The Magellanic clouds are the only galaxies where such an extensive variable star study has been performed, covering variables with a wide range of periods. The total area covered of the LMC by the OGLE-III project is 39.7 square degrees and samples the central regions of the galaxy. In particular, it covers the bar and the inner disc out to a maximum distance of $\sim 5 \mathrm{kpc}$. However, the distribution of OGLE-III fields follows the bar direction from north-west to south-east. Also we note that the size of the LMC is significantly greater than the area covered by OGLE-III (e.g. Saha et al. 2010). Nevertheless, the present data set allows studying the spatial distribution of the different samples of variable stars over the central region of the galaxy, thus tracing the parent population. As a matter of fact, the RR Lyrae stars and the P2Cs are horizontal branch stars tracing the old and predominantly metal-poor population $([\mathrm{Fe} / \mathrm{H}] \lesssim-1 \mathrm{dex})$, while the classical Cepheids trace the young and mainly metalrich $([\mathrm{Fe} / \mathrm{H}] \gtrsim-0.5$ dex $)$ stars. As shown in Fig. 5, the spatial distribution of young stars is concentrated at the centre of the galaxy, nicely tracing the bar region where the star formation occurred at very recent epochs ( $\$ 0.5 \mathrm{Gyr}$ ago $)$. Also, there are two clear overdensities at the end of the bar at $\sim 85$ and 74 degrees. On the other hand, the RR Lyrae stars seem to follow a much broader uniform distribution as is usually observed in other galaxies (Monelli et al. 2010a,b, 2012; de Boer et al. 2011). Interestingly, the ACs do not seem to obviously follow either of the two previous distributions. In particular, while the majority seems concentrated in the innermost regions, where the bulk of the RR Lyrae stars is located, they are not usually placed in the bar. Moreover, there is a clear overabundance in the east direction (RA $\gtrsim 87$ degrees), where the density of both RR Lyrae and Cepheids has significantly decreased. A similar excess does not seem to be present on the other side of the galaxy.

To better compare the spatial distribution of the different class of variable stars, we built the cumulative distributions as a function of the galactocentric radius using the LMC model from van der Marel (2001) to derive the deprojected distance from the centre. They are shown in Fig. 6 for the RR Lyrae stars, classical, P2Cs, and ACs. We have also computed, using a KolmogorovSmirnov test, the probabilities that the various sample are drawn from the same parent population as the RR Lyrae stars. We found that this is $0 \%\left(5 \times 10^{-9} \%\right)$ for classical Cepheids, $0.2 \%$ for $\mathrm{ACs}$, and $87 \%$ for P2C. Therefore, the P2Cs seem to follow the same distribution of the RR Lyrae stars, in agreement with the fact that they both originate in the old population. We have also computed the probability that the ACs come from young classical Cepheids, which is $8 \%$. To avoid possible bias from the area covered by the OGLE-III survey, we repeated these estimations using only subgroups of stars located at distances within 2,3 , and $4 \mathrm{kpc}$ from the LMC centre. We find that the probability that the distributions of the RR Lyrae and P2C coincide is always above $75 \%$, while that of RR Lyrae and classical Cepheids is always below 1\%. In the case of ACs and RR Lyrae stars, the probability widely fluctuates but, with some hint of decreasing probability for increasing the limit radius, ranging from 32 , to 10 , to $0.5 \%$ in the three cases.

These tests do not show any clear similarity between the distributions of ACs with either that of classical Cepheids or RR Lyrae stars. This suggests that they are not uniquely correlated either with the old or with the youngest LMC population.

\section{Discussion of the origin of ACs}

The theoretical scenario that explains the structure, evolution, and pulsation properties is settled for different classes of pulsating variable stars. This has strong implications in the study of the SFH of galaxies, because it is possible to characterise the properties of the parent population (e.g., age and metallicity) by investigating the bright variables associated. However, the presence of ACs does not uniquely identify the nature of their progenitors. The two most favoured formation channels are the evolution of single, metal-poor star with mass $\$ 2 M_{\odot}$, or the evolution of coalescent binary systems of metal-poor stars. Independent of the formation mechanism, a crucial characteristic of the progenitor for producing an $\mathrm{AC}$ appears to be its low metallicity. In fact, central-helium burning structures in the AC mass range, but more metal-rich than $Z \sim 0.0008$ (Gallart et al. 2004a; Fiorentino et al. 2006), are too red to enter the Cepheid instability strip and populate the red clump. To have pulsators at higher metallicity, the mass of the progenitor must be higher. In this case, the star will cross the strip at higher luminosity producing short period classical Cepheids, which ignite the helium quiescently. In particular, the minimum mass of central heliumburning pulsators crossing the instability strip increases from $1.9 M_{\odot}$ (for $Z=0.0004$ ) to $3.6 M_{\odot}$ (for $Z=0.008$, Caputo et al. 2004).

The binary origin is a plausible explanation of ACs having been ubiquitously discovered in low-density, very metal-poor environments ( $Z \leq 0.0008)$, such as nearby purely old dwarf galaxies. In fact, ACs have been observed in dwarf galaxies that do not show any intermediate-age population, such as Draco, Ursa Minor, Sculptor, Sextans, Tucana, LeoII, and Cetus. This implies that, at least in these galaxies, ACs must be the progeny of metal-poor, binary systems. On the other hand, very few systems have been discovered to host both classical and anomalous Cepheids. Gallart et al. (2004b) first pointed out this occurrence in the case of the transition dIrr/dSph galaxy Phoenix, supporting the evidence that the same applies to Sextans A and Leo A. Comparing the spatial distribution of different samples of stars in Phoenix, Gallart et al. (2004b) find that, while classical Cepheids are strongly segregated in the central regions, following the 


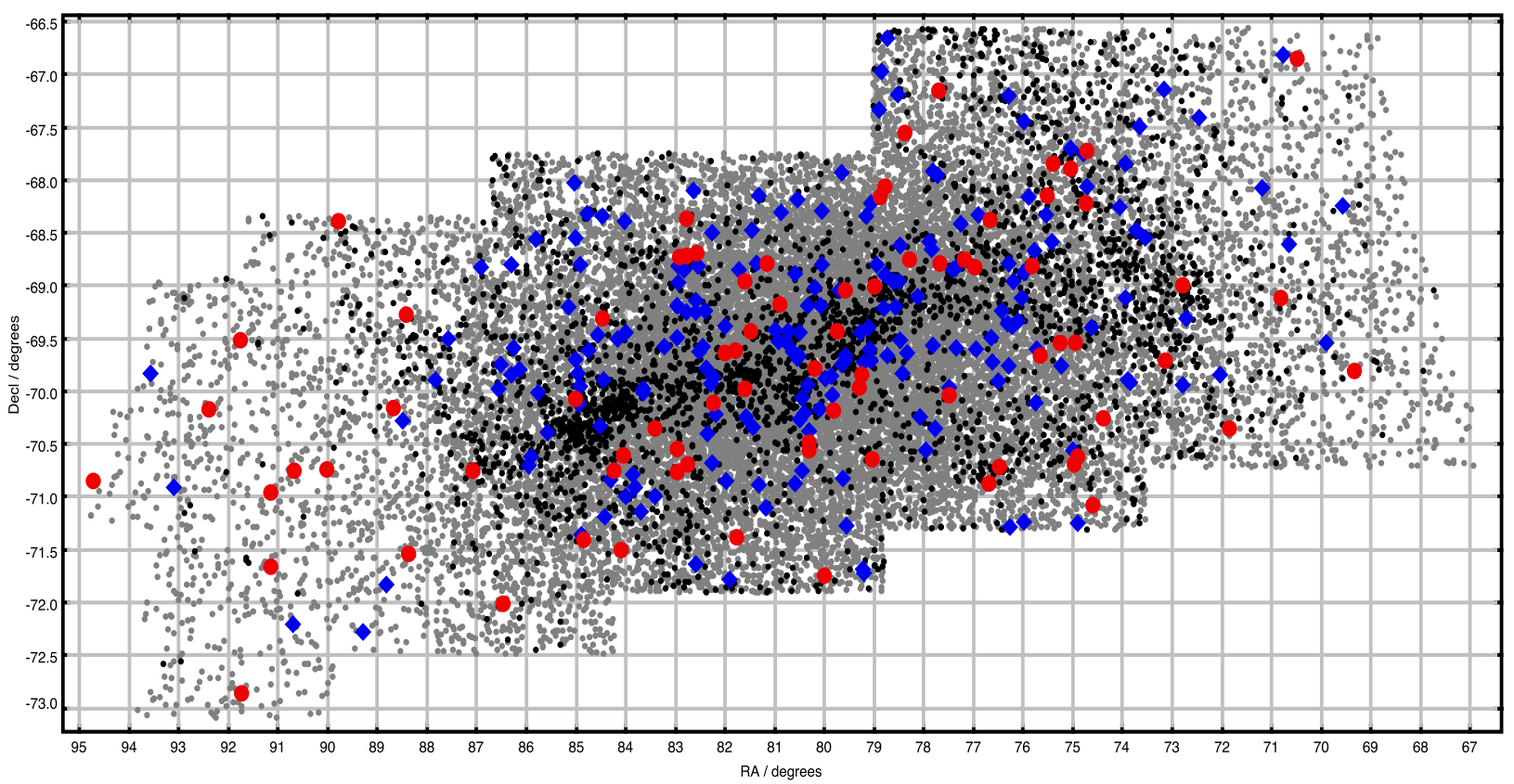

Fig. 5. Spatial distribution of anomalous (red), population II (blue), and classical Cepheids (black), RR Lyrae (grey) in the total area of the Large Magellanic Cloud ( 39.7 square degrees) covered by OGLE III survey.

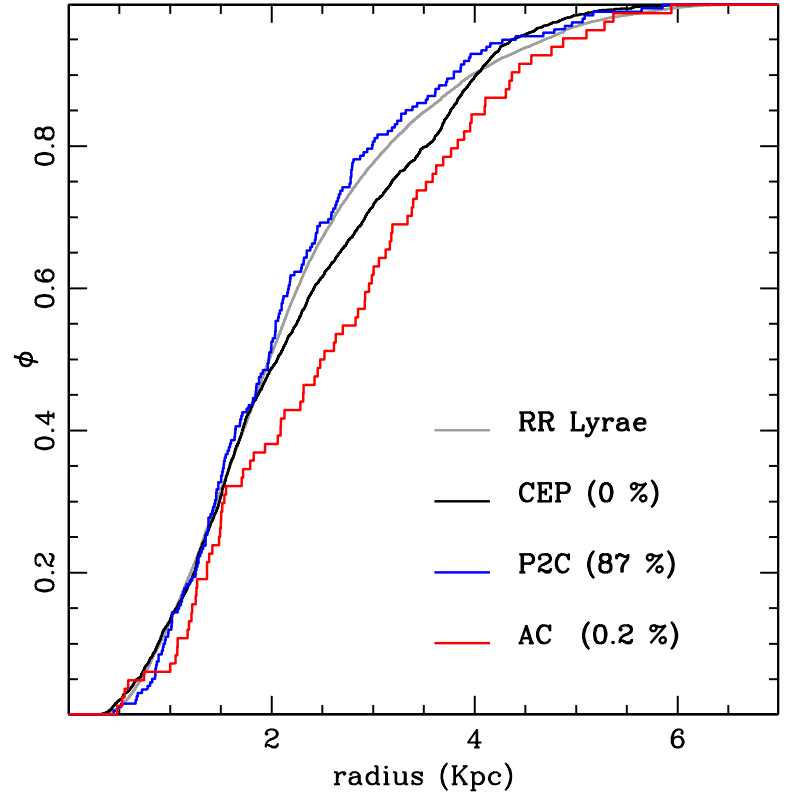

Fig. 6. Cumulative distributions for anomalous (red), population II (blue), and classical Cepheids (black), RR Lyrae (grey).

spatial distribution of the youngest population, the ACs are distributed over a broader area, but are still more concentrated than the RR Lyrae stars, which can be safely taken as representative of the old population. This suggests that the ACs in Phoenix are mostly intermediate-age stars, though the possibility that a few are BSS descendents cannot be excluded. This implies that, despite its prolonged star formation until epochs that are recent enough to produce classical Cepheids, Phoenix experienced little chemical enrichment, in agreement with Hidalgo et al. (2009), and the 1-6 Gyr old population was still metal-poor enough to produce ACs.

\subsection{The case of $L M C$}

The case of the LMC presented here is probably more complex, since the LMC is two orders of magnitude more massive than the typical satellite $\mathrm{dSph}$, and has a complex structure (i.e. disc and bar). Nevertheless, until the work by Soszyński et al. (2008c), no ACs had been unambiguously detected. The present sample of 84 objects is the largest so far for any external galaxy. The coexistence with large samples of different variable stars allowed us to compare the radial distribution of these objects using a Kolmogorov-Smirnov test. We found that the present sample of ACs cannot be associated to the young classical Cepheids. It must be stressed here that given the large sample of AC and classical Cepheids in the LMC, their different nature clearly appears for the first time. Despite ACs occupy the same instability strip of the classical Cepheids as suggested by Caputo et al. (2004) and populate the low-mass and low-metallicity extension, it is clear that the two groups follow different Wesenheit relations (Soszyński et al. 2008c). We also found that the radial distribution of ACs does not correlate with that of the oldest population, as represented by RR Lyrae and P2C, suggesting that, as for of case of Phoenix (Gallart et al. 2004b), a large fraction of single intermediate-age stars is present.

To investigate this hypothesis, we interpreted the properties of ACs in the full picture of the LMC SFH. Given the mass derived in this work (see Sect. 4), which is $1.2 \pm 0.2 M_{\odot}$ for the bulk of ACs, we can estimate the corresponding age. Using scaled solar evolutionary tracks from the $\mathrm{BaSTI}^{2}$ (Pietrinferni et al. 2004) database, we find ages from 1.8 to $5.8 \mathrm{Gyr}$, assuming $Z=0.0006$. However, the age-metallicity relation (AMR), based on spectroscopic analysis, as presented in recent years (Cole et al. 2005; Carrera et al. 2008, 2011), reveals that the mean metallicity in this age range is an order of magnitude greater than the expected maximum value for ACs, being close to $Z=0.008$. Interestingly, the spatial analysis by Carrera et al. (2008) and Carrera et al. (2011) reveals that, in a number of disc fields

2 http://albione.oa-teramo.inaf.it/ 
at different distances from the LMC centre, but also in the bar field from Cole et al. (2005), the metallicity distribution always presents a well-populated tail towards low metallicity ( $Z \sim 0.0004)$, but the most metal-poor stars are systematically older than 10 Gyr. This occurrence seems at odds with our hypothesis that the bulk of ACs are metal-poor intermediate-age stars. However, one could ask whether, due to the limited number of spectroscopic targets so far analysed, we are facing an observational bias, and that very few metal-poor stars exist in the age range from $\sim 2-6 \mathrm{Gyr}$.

We can attempt to estimate the percentage of the mass involved in the star formation episode that generated the ACs, following the simple prescriptions given in Renzini \& Fusi Pecci (1988), which hold for each post-main sequence phase. The number of ACs $\left(N_{\mathrm{AC}}=84\right)$ observed is related to the total luminosity $\left(L_{Z<0.0008}\right)$ of the population they belong to via the following relation: $N_{\mathrm{AC}}=B(t) L_{Z<0.0008} \tau$. Where $B(t)$ is the specific evolutionary flux $\left(\sim 0.15 \times 10^{-10}\right.$ stars per $L_{\odot} \mathrm{yr}^{-1}$, see Renzini \& Fusi Pecci 1988), $\tau$ is the time spent in the instability strip for a mass of about 1.2 $M_{\odot}(\sim 50 \mathrm{Myr}$, see Fiorentino et al. 2006), and $L_{Z<0.0008}$ results to be $1 \times 10^{5} L_{\odot}$. This value is very low related with the total luminosity of the LMC $\left(L_{\mathrm{TOT}}=2 \times 10^{9} L_{\odot}\right)$, even if we account for the limited area covered by the OGLE-III survey. Therefore, the corresponding expected total number of stars from this population is very low, a few thousand over the whole field-of-view, implying that few stars brighter than the Horizonal Branch are expected per square degree. This strongly suggests that such a small event cannot be properly sampled by the SFH analysis or spectroscopic investigation of incomplete samples. This in turn highlights the key role of ACs in this context. In fact, the discovery that a small amount of gas was still poorly enriched 6 Gyr ago gives additional constraints to the SFH that have to be accounted for in the formation and evolution modelling of the LMC.

Finally, we want to briefly comment the possibility that ACs are young, but metal-rich stars looks unfeasible. Stars with metallicity close to $Z=0.008$ are expected to be significantly more massive to enter the instability strip during the central helium-burning phase, with respect of more metal-poor stars. Even if we invoke unusually high mass loss to reconcile with the low-mass we estimated in this work, the mass of the helium core would not change significantly, leaving us with brighter stars, which would be at odds with the observed properties of ACs.

\subsection{Comparison with other galaxies}

ACs appear to be intrinsically rare objects. This is valid in general for nearby dwarf galaxies and for the LMC in particular. The OGLE-III projects surveyed more than 35 million stars, and only 84 ACs were detected. In this case, we can safely assume that the completeness is very high, both because of the large number of measurements and the photometric quality of the data.

Nevertheless, Mateo et al. (1995) first notice that a correlation exists between the frequency of ACs $\left(\log S\right.$, per $\left.10^{5} L_{\odot}\right)$ and the total luminosity of the host galaxy $\left(M_{V}\right)$, valid for many nearby satellites. In particular, it was found that the frequency of ACs decreases for increasing luminosity of the host galaxy. Pritzl et al. (2002) noticed that the surveyed M 31 satellites also follow this trend, as does Phoenix (Gallart et al. 2004b). Figure 7 shows the frequency of ACs as a function of the absolute visual magnitude of the host galaxies. We used the data from Table 5 of Pritzl et al. (2004), to which we added the values for Phoenix (Gallart et al. 2004b), Cetus (Bernard et al. 2009; Monelli et al. 2012), Tucana (Bernard et al. 2009), Andromeda I, and Andromeda III

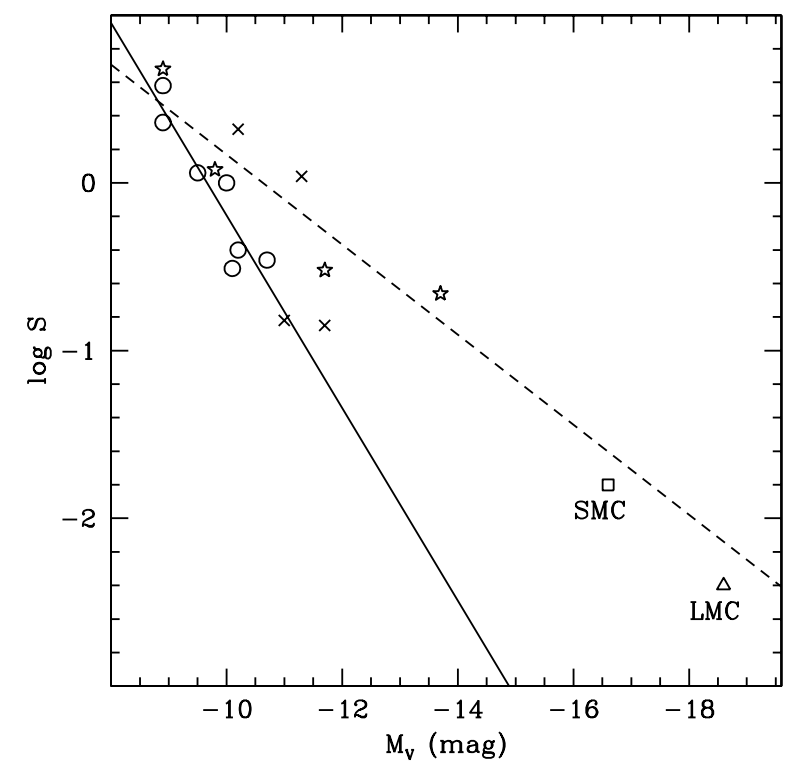

Fig. 7. AC frequency $(\log S)$ per $10^{5} L_{\odot}$ as a function of the absolute $V$ magnitude of the host galaxy $\left(M_{V}\right)$. Open circles show purely old $\mathrm{dSph}$ galaxies, while starred symbols indicate galaxies with large intermediate-age populations. The crosses mark the four M 31 satellites, The LMC and SMC are marked by the open triangle (for an assumed luminosity of $\left.2 \times 10^{9} L_{\odot}\right)^{3}$ and square, respectively. The solid line show the fit to the relation for the old systems only, while the dashed line is the fit to the four crosses.

(Pritzl et al. 2005). The symbols used in the figure differentiate the objects according to their SFH (see the caption for details). In particular, we represent purely old systems (i.e. Ursa Minor, Draco, Sculptor, Leo I, Sextans, Tucana, and Cetus) and dwarf galaxies characterised by an important intermediate-age population (Carina, Fornax, Phoenix, and LeoII). We also plot four M31 satellites (And I, And II, And III, and And VI), for which the data available are not deep enough to set tight constraints on their SFH. In fact, the photometry reaches roughly one mag below the horizontal branch, and it is impossible to clearly detect the presence of a strong intermediate-age population (for example, compare with Carina in Fig. 1 of Harbeck et al. 2001).

The two lines are the fits to the two group of galaxies. The plot suggests that purely old systems and intermediate-age population systems follow different relations. In particular, when an intermediate-age population is present, the frequency of ACs tends to be higher than expected from a purely old population, and the discrepancy increases for increasing luminosity. The underlying physical mechanism of such a relation is unclear. However we only expect ACs resulting from the evolution of binary systems in purely old systems. Thus, the correlation with the luminosity, hence the mass, of the host galaxy may indicate that binary systems have higher chances of surviving in low-mass systems. It is worth recalling in this context that a similar correlation has been found for BSSs. In fact, Momany et al. (2007) have first shown that the frequency of BSS with respect of the number of horizontal branch stars decreases for increasing luminosity of the host galaxy. This might be an independent evidence that an evolutionary link exists between the binary systems, BSSs, and ACs. If such a correlation could be

3 The present value for the LMC is a lower limit, since ACs have so far been detected only in the central region of the LMC. However, the LMC is known to be significantly larger, possibly occupying an area \% times larger (see e.g. Irwin 1991; Majewski et al. 2009; Saha et al. 2010). 
explained this way for purely old systems, the fact that galaxies known to have intermediate-age population present an excess of ACs, compared to purely old galaxies, would be simply due to the coexistence of ACs formed via both formation channels. Among the four M31 satellites, two follow the fit of the old dSphs (And II, And III), while two present an apparent excess of ACs (And I, And VI). This is possibly because the small fraction of these galaxies covered by the data for these galaxies introduces some bias, but it is also possible that And I and And VI host some intermediate-age population, at present undetected but responsible for this effect.

What seems really surprising is that both Magellanic clouds appear to follow the same relation of these four galaxies, but in a significantly higher luminosity regime. It is also interesting to note that the SMC hosts very few ACs candidates (3 F and 3 FO Soszyñski et al. 2010). As for to the LMC, it presents an excess of ACs, thus supporting that it is possible that a small population of intermediate but metal-poor stars exists.

The new OGLE-IV survey will possibly shed light on this problem. In fact, it is well known that a significant age gradient is present in the disc of the LMC (Gallart et al. 2008; Carrera et al. 2011) in the sense that the age of the youngest population gets older for increasing radius. This means that, starting from a certain distance, we do not expect any population that is young enough to produce ACs, thus leaving only the BSS aftermath, if present. Moreover, the absence of young main sequence stars will permit direct detection of BSSs, thus allowing a direct correlation with ACs. The distance where this is expected to occur is far beyond the present coverage of the OGLE-III survey, but it will be definitely covered by OGLE-IV, at present in execution. Stronger constraints on the formation mechanisms of the ACs in the LMC are therefore expected in the near future. Also, a systematic investigation of the M31 satellites to derive the details of their SFH, which is at present perfectly feasible for the ACS and WFC3 cameras on-board the Hubble Space Telescope, would help solve this problem.

\section{Conclusions}

We have analysed the pulsation and spatial properties of 83 ACs discovered by the OGLE-III survey in the LMC. The main results obtained are the following:

- We classified one star (V-166), previously identified as P2Cs, as bona-fide ACs, thus increasing this total number to 84 stars.

- We performed the Fourier analysis of the $V$-band data, deriving the $V$-band amplitude $A_{V}$ and mean $V$ values for the 83 stars for which the $V$ photometry is available, with only one excluded (V-82). We then applied the method introduced by Marconi et al. (2004) to simultaneously derive individual masses and pulsation modes. We find good agreement with the OGLE-III classification for most of the stars $(\sim 85 \%)$, based on the Wesenheit relation and the morphology of light curves. The mass of the ACs ranges from 0.8 to $1.8 M_{\odot}$, with a mean value of $1.2 \pm 0.2 M_{\odot}$.

- The large sample of ACs allowed, for the first time, to unambiguously show that anomalous and classical Cepheids are different. In fact, even though they populate the same instability strip, they follow distinct period-luminosity relations and different spatial distributions.

- We compared the spatial distribution of the ACs with that of other samples of variables (RR Lyrae, P2C, classical Cepheids) using a Kolmogorov-Smirnov statistical test. We found that RR Lyrae and $\mathrm{P} 2 \mathrm{C}$, both bona-fide representatives of the old population $(>10 \mathrm{Gyr})$ follow the same distribution, which is different from that of the young classical Cepheids. The distribution of ACs does not resemble any of the other samples. We conclude that a large fraction of the ACs descend from single, metal-poor, intermediate-age stars that are 1-6 Gyr old.

- The comparison with the presently available SFH and AMR shows that such a population is negligible. We show that the total mass formed in that star formation event is about $5 \times$ $10^{-5}$ the total mass of the LMC, thus resulting in very few stars that are very unlikely to be observed in spectroscopic surveys or in statistical approaches to derive the SFH. This shows the importance of variable stars and rare objects like ACs for giving independent constraints on the modelling of galaxy evolution.

- We reanalysed the frequency-luminosity relation discovered by Mateo et al. (1995), showing that, if only purely old systems are considered, the correlation is tighter and steeper. The objects that present important intermediate-age population tend to larger AC frequency, suggesting that ACs formed from single stars are also present.

The detection and characterisation of ACs at much greater distances from the LMC centre would be very interesting. In fact, given the strong gradients in the LMC stellar population, starting from $\approx 8 \mathrm{Kpc}$ the young population completely disappears. The detection of ACs in these regions of the LMC would strongly point to the binary origin.

Acknowledgements. We sincerely thank the anonymous referee for his/her pertinent comments that improved the readability of this paper. We warmly thank Igor Soszyński for sharing the individual $V$-band photometry; Eline Tolstoy, Giuseppe Greco, Marcella Marconi, Michele Bellazzini, and Ricardo Carrera are warmly thanked for useful discussions and support. Carme Gallart, Edouard Bernard, Giuseppe Bono, and Santi Cassisi are appreciated for reading an early draft of this manuscript. G.F. has been supported by the INAF fellowship 2009 grant. M.M. acknowledges the support by the IAC (grant 310394), the Education and Science Ministry of Spain (grants AYA2007-3E3506, and AYA2010-16717).

\section{References}

Arellano Ferro, A., Giridhar, S., Rojas López, V., et al. 2008, Rev. Mex. Astron. Astrofis., 44, 365

Baldacci, L., Rizzi, L., Clementini, G., \& Held, E. V. 2005, A\&A, 431, 1189

Bernard, E. J., Monelli, M., Gallart, C., et al. 2009, ApJ, 699, 1742

Bersier, D., \& Wood, P. R. 2002, AJ, 123, 840

Bono, G., Caputo, F., Santolamazza, P., Cassisi, S., \& Piersimoni, A. 1997, AJ, 113, 2209

Bono, G., Groenewegen, M. A. T., Marconi, M., \& Caputo, F. 2002, ApJ, 574, L33

Caputo, F., Cassisi, S., Castellani, M., Marconi, G., \& Santolamazza, P. 1999, AJ, 117, 2199

Caputo, F., Castellani, V., Degl'Innocenti, S., Fiorentino, G., \& Marconi, M. 2004, A\&A, 424, 927

Cardelli, J. A., Clayton, G. C., \& Mathis, J. S. 1989, ApJ, 345, 245

Carrera, R., Gallart, C., Hardy, E., Aparicio, A., \& Zinn, R. 2008, AJ, 135, 836

Carrera, R., Gallart, C., Aparicio, A., \& Hardy, E. 2011, AJ, 142, 61

Cassisi, S., \& Salaris, M. 2011, ApJ, 728, L43

Castellani, V., \& degl'Innocenti, S. 1995, A\&A, 298, 827

Catelan, M., \& Cortés, C. 2008, ApJ, 676, L135

Cioni, M.-R. L., Clementini, G., Girardi, L., et al. 2011, A\&A, 527, A116

Cole, A. A., Tolstoy, E., Gallagher, III, J. S., \& Smecker-Hane, T. A. 2005, AJ, 129,1465

Corwin, T. M., Carney, B. W., \& Nifong, B. G. 1999, AJ, 118, 2875

Dall'Ora, M., Ripepi, V., Caputo, F., et al. 2003, AJ, 126, 197

de Boer, T. J. L., Tolstoy, E., Saha, A., et al. 2011, A\&A, 528, A119

Demarque, P., \& Hirshfeld, A. W. 1975, ApJ, 202, 346

Di Criscienzo, M., Caputo, F., Marconi, M., \& Cassisi, S. 2007, A\&A, 471, 893

Dolphin, A. E., Saha, A., Claver, J., et al. 2002, AJ, 123, 3154

Fiorentino, G., Limongi, M., Caputo, F., \& Marconi, M. 2006, A\&A, 460, 155 
Fiorentino, G., Contreras Ramos, R., Clementini, G., et al. 2010, ApJ, 711, 808 Fiorentino, G., Tolstoy, E., Diolaiti, E., et al. 2011, A\&A, 535, A63

Fiorentino, G., Contreras Ramos, R., Tolstoy, E., Clementini, G., \& Saha, A. 2012, A\&A, 539, A138

Gallart, C., Aparicio, A., Freedman, W. L., et al. 2004a, AJ, 127, 1486

Gallart, C., Stetson, P. B., Hardy, E., Pont, F., \& Zinn, R. 2004b, ApJ, 614, L109

Gallart, C., Stetson, P. B., Meschin, I. P., Pont, F., \& Hardy, E. 2008, ApJ, 682, L89

Harbeck, D., Grebel, E. K., Holtzman, J., et al. 2001, AJ, 122, 3092

Harris, J., \& Zaritsky, D. 2009, AJ, 138, 1243

Harris, H. C., Silberman, N. A., \& Smith, H. A. 1998, in A Half Century of Stellar Pulsation Interpretation, ed. P. A. Bradley, \& J. A. Guzik (San Francisco: ASP), ASP Conf. Ser., 135, 164

Haschke, R., Grebel, E. K., \& Duffau, S. 2011, AJ, 141, 158

Hidalgo, S. L., Aparicio, A., Martínez-Delgado, D., \& Gallart, C. 2009, ApJ, 705,704

Hodge, P. W., \& Wright, F. W. 1978, AJ, 83, 228

Hoessel, J. G., Saha, A., Krist, J., \& Danielson, G. E. 1994, AJ, 108, 645

Irwin, M. J. 1991, in The Magellanic Clouds, ed. R. Haynes, \& D. Milne (Paris: IAU), IAU Symp., 148, 453

Kaluzny, J., Kubiak, M., Szymanski, M., et al. 1995, A\&AS, 112, 407

Kinemuchi, K., Harris, H. C., Smith, H. A., et al. 2008, AJ, 136, 1921

Kuehn, C., Kinemuchi, K., Ripepi, V., et al. 2008, ApJ, 674, L81

Kuehn, C. A., Smith, H. A., Catelan, M., et al. 2011, AJ, 142, 107

Majewski, S. R., Nidever, D. L., Muñoz, R. R., et al. 2009, in IAU Symp. 256, ed. J. T. van Loon, \& J. M. Oliveira (Paris: IAU), 51

Marconi, M., Fiorentino, G., \& Caputo, F. 2004, A\&A, 417, 1101

Mateo, M., Fischer, P., \& Krzeminski, W. 1995, AJ, 110, 2166

Momany, Y., Held, E. V., Saviane, I., et al. 2007, A\&A, 468, 973

Monelli, M., Gallart, C., Hidalgo, S. L., et al. 2010a, ApJ, 722, 1864
Monelli, M., Hidalgo, S. L., Stetson, P. B., et al. 2010b, ApJ, 720, 1225 Monelli, M., Cassisi, S., Mapelli, M., et al. 2012, ApJ, 744, 157

Nemec, J. M., Wehlau, A., \& Mendes de Oliveira, C. 1988, AJ, 96, 528 Norris, J., \& Zinn, R. 1975, ApJ, 202, 335

Pietrinferni, A., Cassisi, S., Salaris, M., \& Castelli, F. 2004, ApJ, 612, 168 Pietrzyński, G., Thompson, I. B., Gieren, W., et al. 2010, Nature, 468, 542 Pietrzynski, G., Thompson, I., Graczyk., D., et al. 2011, ApJ, 742, L20 Pritzl, B. J., Armandroff, T. E., Jacoby, G. H., \& Da Costa, G. S. 2002, AJ, 124, 1464

Pritzl, B. J., Armandroff, T. E., Jacoby, G. H., \& Da Costa, G. S. 2004, AJ, 127, 318

Pritzl, B. J., Armandroff, T. E., Jacoby, G. H., \& Da Costa, G. S. 2005, AJ, 129, 2232

Reid, W. A., \& Parker, Q. A. 2010, MNRAS, 405, 1349

Renzini, A., \& Fusi Pecci, F. 1988, ARA\&A, 26, 199

Renzini, A., Mengel, J. G., \& Sweigart, A. V. 1977, A\&A, 56, 369

Romaniello, M., Salaris, M., Cassisi, S., \& Panagia, N. 2000, ApJ, 530, 738

Saha, A., Olszewski, E. W., Brondel, B., et al. 2010, AJ, 140, 1719

Siegel, M. H., \& Majewski, S. R. 2000, AJ, 120, 284

Sills, A., Karakas, A., \& Lattanzio, J. 2009, ApJ, 692, 1411

Smith, H. A., \& Stryker, L. L. 1986, AJ, 92, 328

Soszyński, I., Poleski, R., Udalski, A., et al. 2008a, Acta Astron., 58, 153

Soszyński, I., Poleski, R., Udalski, A., et al. 2008b, Acta Astron., 58, 163

Soszyński, I., Udalski, A., Szymański, M. K., et al. 2008c, Acta Astron., 58, 293

Soszyński, I., Udalski, A., Szymański, M. K., et al. 2009a, Acta Astron., 59, 1

Soszyński, I., Udalski, A., Szymański, M. K., et al. 2009b, Acta Astron., 59, 239

Soszyñski, I., Poleski, R., Udalski, A., et al. 2010, Acta Astron., 60, 17

Udalski, A., Soszyński, I., Szymanski, M. K., et al. 2008, Acta Astron., 58, 89

van der Marel, R. P. 2001, AJ, 122, 1827

Zinn, R., \& Dahn, C. C. 1976, AJ, 81, 527 